\title{
Relation between Helicobacter Pylori infection and Irritable Bowel Syndrome
}

\author{
Ali Taha Ali, Yasser Mohamed kamal, Romany mekhael kerollos, \\ Department of internal medicine, Sohag University
}

\begin{abstract} controversial. syndrome

Those groups will be classified according to severity

Also the affected groups will be classified according to subtypes

All patents underlying the following:

(1) history will be taken including:

(2)Full clinical examination

(3) Laboratory investigations

- Fasting blood glucose level,HBA1C

- renal function .liver function, complete blood counting

- Abdominal ultrasonography

- H.pylori stool antigen test titr (SAT)
\end{abstract}

Background: Irritable bowel syndrome (IBS) is a chronic functional gastrointestinal disorder. The association of Helicobacter pylori(H. pylori) infection with IBS still remains

Objective: The objective of this study is to determine the rate of H. pylori infection among patients with IBS through the detection of H. pyloriStool Antigen Teesttitre (SAT)

Materials \&Methods :, This study will be performed on 80 patient attended GIT out patient clinic of sohag university hospital from 6/2017to 3/2018 will be classified into:

1-controle group (20patients)who do not fulfill criteria for diagnosis of irritable bowel

2-Affected group(60patients) who fulfill criteria for diagnosis of irritable bowel syndrome

Results: Comparison was done among all types of IBS as regard H.Pylori SAT revealed constipation predominant cases have the highest number(33\%) of H.Pylori SAT positive cases while lowest number of cases(4\%) were unclassified, Comparison was done among all grades of IBS as regard H. Pylori SAT revealed grade 3 cases the highest number of H. Pylori SAT positive cases, lowest number of cases were grade 1

Conclusion: In this study revealed positive relationship between $\mathrm{H}$. Pylori infection and irritable bowel syndrome as regard types of irritable bowel syndrome, the incidence of $\mathrm{H}$. Pylori infection was more with constipation predominant patients and as regard severity of irritable bowel syndrome, $\mathrm{H}$. Pylori infection more common in grade 3

Keywords :Irritable bowel syndromeHelicobacter pylori infection

\section{Introduction}

(H. pylori) is a gram-negative, microaerophilic spiral bacterium found usually in the stomach; it causes chronic gastritis and gastric ulcers, and has been linked to the development of duodenal ulcers and stomach studies have linked $\mathrm{H}$. pyloriinfection with a wide range of extra gastric diseases Irritable bowel syndrome (IBS) is a chronic functional gastrointestinal disorder that is characterized by abdominal pain and changes in stool habits. Several studies have described the role of various risk factors in the pathogenesis of IBS, including different infections such as $H$. pylori infection. However, other studies have denied such associationThe induction of typical abdominal discomfort associated with IBS occurs predominantly in $\mathrm{H}$. pylori infected patients, indicating that $H$. pylori infection may be involved in triggering visceral hypersensitivity in patients 
with IBSIn contrast, several studies have reported that there is no association between $\mathrm{H}$. pyloriinfection and IBSHence, it has been suggested that with the exception of unexplained iron deficiency anemia and idiopathic thrombocytopenic purpura, H. pylori infection has no proven role in other extra intestinal diseases

\section{MATERIALS \&METHODS :}

This study will be performed on 80 patient attended GI Tout patient clinic of sha university hospital from 6/2017to 3/2018 will be classified into1-controle group (20patients)who do not fulfill criteria for diagnosis of irritable bowesyndrom2Affectgroup(60patients) who fulfill criteria for diagnosis of irritable bowel syndrome Those groups will be classified according to severity into* Grade1mild (awareness of the symptom but easily tolerated);

*Grade2 relevant (interference withnormal

activity);*Grade3severe(incapacitating )Also the affected groups will be classified according to subtypes:

1-constipation predominant

2-diarrhea predominant

3-mixed

4-unclassified

All patents underlying the following

(1) history will be taken including:

Age, Sex,drug history

*History of abdominal pain : criteria, severity ,aggravating factors, releasing factors*history of diarrhea and or constipation associated with abdominal pain or not

(2) Examination:

(3) Laboratory investigations

- Fasting blood glucose level,HBA1C

- renal function .liver function, complete blood counting

- Abdominal ultrasonography

*Inclusion criteria:

- All of the patients had been diagnosed and treated for IBS

* Exclusion Criteria. 1-who had recently undergone digestive surgery.

2-IntakeofNSAIDs ,immunosuppressivedrugsorantibioti csin the previous 3 months

3-Major psychiatric disease

4-Excessive alcohol consumption

5-Rrenal or Hepatic disease

Stool antigen test (SAT) is a noninvasive method with good sensitivity and specificity, 94\% and $97 \%$ respectively in global me analysis, in the diagnosis of $\mathrm{H}$. pylori infection. This method detects the presence of $\mathrm{H}$. pylori antigen in stool samples. The accuracy of SAT is influenced by several factors, like antibiotic, PPI, N-acetyl cysteine, bowel movement and upper gastrointestinal bleeding. Preservation of the specimen, like temperature and transport time before testing, and cutoff valve also have impacts on diagnostic accuracy of SATEIA(EnzymeImmuonoAssay)Te st kitEIA test kit used in this study used in Acon laboratories in USA The H.pylori antigen EIAtest is an enzyme immunoassay for the qualitative and quantitative detection in human stool .itis intended as an aid in the diagnosis of possible H.pylori infection and in the follow up of patients undergoing anti microbial therapy

Princible:The H.pylori antigen EIA test kit is asolid enzyme immunoassay based on sandwich principle for the qualitative and quantitative detection of H.pylori antigen in human stool the microwell plate is coated with anti H.pylori anti bodies. During testing the antigens are extracted out with extraction solution and added to the antibodies to H.pyloriand then incubated if the specimenscontain h.pylori antigens

:it will bind to anti body coated on the microwell plate and simultaneously bind to the conjugate to form immobilized antibody - H.pylori 
antigen conjugate complexes if the specimen donot contain H.pylori stool antigens, the complexes will not be formed. After initial incubation, the micro well plate is washed to remove un bound materials. substrate $\mathrm{A}$ and are added and then incubated to produce blue color indicating the amount of H.pylori antigen present in the specimens .sulfuric acid solution is added to the microwell plate to stop the reaction producing color change from blue to yellow.the color intensity which correspond to the amount of $\mathrm{H}$. pylori antigens present in the specimens, it is measured with microplate reader at $450 / 630-700$ nmor 450nm

\author{
Interpretation of results: \\ *Qualitative Index value: \\ Positive $>1.1$ \\ Negative $<0.9$ \\ Equivocal (0.9--1.1) \\ *Quantitative index value: \\ Positive $>0.055$ \\ Negative $<0.045$ \\ Equivocal ( 0.055--0.045 \\ Performance characteristics \\ 1 -sensitivity and specificity : \\ The H.pylori antigen EIA test kit \\ habeen compared to a \\ leadingcommercialH.pylori EIA test \\ using clinical specimen, the results \\ showthat the clinical sensitivity \\ ofH.pylori is $98.6 \%$ and specificity is \\ $95.4 \%$
}

\section{Results}

Our study on 80 patients including 27 female 53 male.ranging from 20-60 years old,after complete history taking and full clinical examination of the cases in our study we found that $17.5 \%$ of those patients donot suffer from irritable bowel syndrome $82.5 \%$ were complaining from irritable bowel syndrome,IBS patients were classified according to type of IBS And about 33patients was constipation predominant $(41 \%)$, about 24 patients were diarrhea predominant (30\%) ,about 5patients were mixed (6.25\%) , and 4 patient were un classified (5\%) Those patients were classified also according to severity of IBS About 32patient $(40 \%)$ were suffering from mild degree(awareness of the symptom but easily tolerated) about 21patient (26.25\%)moderate degree; relevant (interference with normal activity); and about 13patients (16.25\%)severe (incapacitating) Stool antigen test titre(SAT) was done for all studied cases and about 43 patients $(53.75 \%)$ were SAT positive and about 37 patients $(46.25 \%)$ were SAT negativeDistribution of studied population according to H.Pylori SAT 46\% SAT negative,53.7\% sat positiveComparison was done among all types of IBS as regard H.Pylori SAT revealed constipation predominant cases have the highest number(33\%) of H.Pylori SAT positive cases while lowest number of cases(4\%) were unclassifiedComparison was done among all grades of IBS as regard H.Pylori SAT revealed grade 3 cases thethe highest number of H.Pylori SAT positive cases,lowest number of cases were grade 1 
Table4: Comparison among types of IBS as regard H. pylori SAT

\begin{tabular}{|c|c|c|}
\hline Types of IBS & $\begin{array}{c}\text { Mean } \pm \text { SD } \\
\text { Median (range) }\end{array}$ & P value \\
\hline No IBS & $0.11 \pm 0.15$ & \multirow{2}{*}{0.0007} \\
& $0.1(0-0.36)$ & \\
\hline Type C & $0.20 \pm 0.22$ & \\
& $0.13(0-0.9)$ & \\
Type D & $0.12 \pm 0.15$ & \\
& $0.03(0-0.5)$ & \\
\hline Type M & $0.11 \pm 0.15$ & \\
& $0.1(0-0.36)$ & \\
\hline \multicolumn{2}{|c|}{ Type U } & 0 \\
\hline p1 $=0.0001, \mathrm{p} 2=0.01, \mathrm{p} 3=0.05, \mathrm{p} 4=1.00, \mathrm{p} 5=0.10, \mathrm{p} 6=0.36$, \\
$\mathrm{p} 7=0.01, \mathrm{p} 8=0.82, \mathrm{p} 9=0.12, \mathrm{p} 10=0.14$ \\
\hline
\end{tabular}

(p1compared no IBS with type C, p2compared no IBS with type D,p3compared no IBS with type M,p4compared no IBS with type U, p5 compared with type C \&D, p6compared with type C \&M, p7compared with type $C \& U, p 8$ compared with type D\&M, p9 compared with type D\&U, p10 compared with type $M$ )

Comparison among types of IBS as regard $\mathrm{H}$. pylori SAT

\begin{tabular}{|c|c|c|c|}
\hline Number (\%) & $\begin{array}{c}\text { H. pylori SAT } \\
\text { Negative }\end{array}$ & $\begin{array}{l}\begin{array}{l}\text { H. pylori SAT } \\
\text { Positive }\end{array} \\
\end{array}$ & \multirow[t]{2}{*}{$P$ value } \\
\hline () & Number (\%) & Number (\%) & \\
\hline No IBS & $14(100 \%)$ & 0 & \multirow{5}{*}{$<0.0001$} \\
\hline Type C & $11(33.33 \%)$ & $22(66.67 \%)$ & \\
\hline Type D & $12(50.00 \%)$ & $12(50.00 \%)$ & \\
\hline Type M & $2(40.00 \%)$ & $3(60.00 \%)$ & \\
\hline Type U & $4(100 \%)$ & 0 & \\
\hline
\end{tabular}

(p1compared no IBS with type C, p2compared no IBS with type D,p3compared no IBS with type

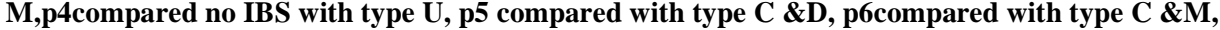
p7compared with type $C \& U$, p8 compared with type D\&M, p9 compared with type D\&U, p10 compared with type $M \& U$ )

Comparison among grades of IBS as regard H. pylori:

\begin{tabular}{|c|c|c|}
\hline Grades of IBS & $\begin{array}{c}\text { Mean } \pm \text { SD } \\
\text { Median (range) }\end{array}$ & P value \\
\hline No IBS & 0 & \multirow{4}{*}{0.0001} \\
\hline Grade 1 & $\begin{array}{c}0.08 \pm 0.09 \\
0.07(0-0.4)\end{array}$ & \\
\hline Grade 2 & $\begin{array}{c}0.14 \pm 0.18 \\
0(0-0.5)\end{array}$ & \\
\hline Grade 3 & $\begin{array}{c}0.35 \pm 0.25 \\
0.45(0-0.9)\end{array}$ & \\
\hline $\mathrm{p} 1=0.0008, \mathrm{p} 2=0.03$ & $07, \mathrm{p} 4=0.77, \mathrm{p} 5$ & $\mathrm{p} 6=0.01$ \\
\hline
\end{tabular}

(p1compared no IBS with grade1, p2compared no IBS with grade2,p3compared no IBS with grade3, p4compared grade $1 \& 2$, p5 compared with grade $1 \& 3$, p6compared with grade $2 \& 3$ )

\section{Discussion}

IBS is a substantial medical challenge to society, and the development of a novel treatment for this disease is frustrated by the lack of insight into its etiology and pathogenesis. In the present study we explored the potential association between $H$. pylori and IBS.Previous basic studies have suggested that the systemic inflammation provoked by CagA (cytotoxin-associated gene A) and VacA (vacuolatingcytotoxin) of $H$. pylori may link this bacterium to the pathogenesis of IBS (1)

starting to test this hypothesis through a cross sectionalanalysis of the effects of $H$. pylori infection inpatientpopulation attending Sohage University Hospital internal medicine outpatient clinic and gastro intestinal 
tract out patientclinic.Numerous obstacles hamper the study of therelation between $H$ pylori infection and IBS. Most cases of $H$ pylori infection are silent and, although abdominal symptoms are commonplace, not all dyspeptic patients seek medical advice. $(2,3)$

Symptom assessments must be meticulous as $H$ pylori infection may induce someabdominal symptoms while other symptoms

may not be associated with the infection.Moreover, to assess the association between H.pyloriinfection and IBS, $H$ pylori relatedorganic disease must be ruled out. Whereas the Sensitivity of the SAT used in this study was high, the specificity was relatively low. The low specificity would, however, mask a possible effect of $H$ pylori on gastrointestinal symptomatology as symptoms caused by $H$ pylori may also be reported by people who tested SAT. The diagnostic accuracy of commercially available serology kits varies considerably between different laboratories(4)

The specificity of the present assay, however, was only slightly lower than that which could be obtained with commercially available detection kits in this study population

The main aspect in our study is to detect the pattern of H.pylori infection among patients with irritable bowel syndrome And so we made comparison between those without and with IBS according to H. pylori SAT and we found that big partition of IBS cases were SAT positive (65\%) and 35\% were SAT negative and among patients without IBS criteria $30 \%$ of cases wereSAT positive this was near the results of many studies like (Ford AC , Moayyedi P, Jarbol DE et al. Meta-analysis: $H$ elicobacter pylori 'test and treat' compared with empirical acid suppression for managingdyspepsia . Aliment PharmacolTher2008 ; 28 : 534 - 44 .)revealed only $50 \%$ were
SAT positive and $50 \%$ were SAT negativeinfected patints this may be explained by difference in dietary habits as we mentioned abovesocioeconomic state as this study revealed that constipation presomimantDifferences between both studies this may may be Explained by different number of cases and change in locality as H.Pylori infection is more common in local countries like Egypt as H.Pyloriis more prevalent among older adults, African, and lower socioeconomic groups as the main route of transmission of this bacterium is fecooral route so bad hygiene and and low socioeconomic state in our locality affect morbidity of infection by this bacterium.

so we should mention that Rovell and Ford conducted meta-analysis on the study of the epidemiology of IBS(RovellRm,FordAc,globalprevale nceand risk factors for irritable bowel syndrome ametaanalysis clin ,gastroenterol hepatol201210:72-721)

the study revealed that socioeconomic state affect the prevalence of IBS,this study was done on 300patent suffering from manifestations of irritable bowel syndrome and fulfilling the Rome criteria for its diagnosis ,those patients of various socioeconomic state and various locality ,upper endoscpy and tissue biopsy was done for those patients .percentage of h.pylori positive patints was more among low socioeconomic state patients Another item which is important In description of the pattern of H.pylori infection among patients with irritable bowel syndrome is to compare different types of IBS according to severity of H.pylori infection which can be detected by stool antigen titer our study revealed constipation predominant IBS have the highest records of H.pylori SAT(41\%)then diarrhea predominant have the $2^{\text {nd }}$ 
level(30\%) while mixed and unclassified cases have the lowest records this results was near many studies like (McCune A, Lane A, Murray $\mathrm{L}$ et al. Reduced risk of atopic disorders in adults with Helicobacter pylori infection . Eur J GastroenterolHepatol 2013 ; 15: 637

-40)which revealed that the two most common subtypes are diarrhea predominant $(45 \%)$ and constipation predominant $(35 \%)$ but the difference from this study is that the most common subtype is diarrhea predominant not constipation predominant as in our study but unclassified and mixed have higher results and may be explained by deficient number of these cases in my study and affected by dietary habits psychical treatment ,support and follow up which is deficient in our locality $(5,6)$

according( Yuka,Yomatakaetal) IBS, and its subtypes prevalence is affected by age sex ,bowel habits, ibs may be more common among H.Pylori infected patints this may be explained by difference in dietary habits as we mentioned abov

socioeconomic state as this study revealed that constipation predominant Differences between both studies this may may be Explained by different number of cases and change in locality as H.Pylori infection is more common in local countries like Egypt as H.Pyloriis more prevalent among older adults, African, and lower socioeconomic groups as the main route of transmission of this bacterium is fecooral route so bad hygiene and and low socioeconomic state in our locality affect morbidity of infection by this bacterium.

so we should mention that Rovell and Ford conducted meta-analysis on the study of the epidemiology of IBS(RovellRm,FordAc,globalprevale nceand risk factors for irritable bowel analysis clin ,gastroenterol hepatol201210:72-721)syndrome

ameta

by difference in dietary habits as we mentioned above

we should mention in (a meta analysisHalverson et al) reports that the prevalence of IBS deceased after increasd years with acute gastroenteritis as post infectios IBS incidence is increasing after gastroenteritis and has higher prevalence $(\mathbf{7 , 8 , 9 )}$

The last aspect we want to explore in our study is to compare severity of IBS with the severity of H.pylori infection and we found that there highest records ofH.pylori SAT was grade 111 sever the lowest recordswas grade 1 this was like many results like(10)

It is interesting to observe how patients with visceralhypersensitivity also exhibit $H$. pylori infections. This factsuggests that this bacteriummay be involved in triggering abdominal painin IBS patients .(11)

Such association between $H$. pylori and IBS can be partially explained by the presence of different types of $H$. pylorivirulence factors, in addition to host genetic predisposition and environmental factors; moreover, the clinical outcomes are determined by the interplay of these factors $(\mathbf{1 2 , 1 3})$

Conclusion In this study revealed positive relationship between $\mathrm{H}$. Pylori infection and irritable bowel syndrome as regard types of irritable bowel syndrome, the incidence of $\mathrm{H}$. Pylori infection was more with constipation predominant patients and as regard severity of irritable bowel syndrome, $\mathrm{H}$. Pylori infection more common in grade 3

Recommendation This study affected by limited number of cases, specificity and sensitivity of SAT ELISA APPARATUS, more researches are need to be done to explore this relation , and to explore the effect of $\mathrm{H}$. Pylori 
eradication therapy on improvement of symptoms of irritable bowel syndrome which may help in adding TTT of $\mathrm{H}$. Pylori infection as a line of management of IBS

\section{References:}

1-. Hwaid AH, Hasan ASH, Al -Duliami AA, Al-MarjaniMF. Seroprevalence of Helicobacter pylori infection and its relation to abo/rhesus blood groups in Diyala, Iraq. International Journal of Current Research. 2013;5(12):4268-71.

2- Nguyen TN, Barkun AN, Fallone CA, Host determinants of Helicobacter pylori infection and its clinical outcome. Helicobacter. 1999;4(3): 18597.

3-.Basso D, Plebani M. H. pylori infection: bacterial virulence factors and cytokine gene polymorphisms as determinants of infection 2004;41(3):31-37.

4-.Posselt G, Backert S, Wessle RS. The functional interplay of Helicobacter pylori factors with gastric epithelial cells induces a multi-step process in pathogenesis. Cell Commun Signal. 2013;11:77. 26.Kalali B,Mejías-Luque R, Javaheri A, Gerhard M. H. pylori virulence factors: influence on immune system and pathology.Mediators of Inflammation. 2014;2014:426309.
5-.Shiota S, Suzuki R, Yamaoka Y. The significanceof virulence factors in Helicobacter pylori. J Dig Dis.2013;14(7):341-9.

6-.Sgouras DN, Trang TT, Yamaoka Y. Pathogenesis of Helicobacter pylori infection. Helicobacter. 2015;20 Suppl $1: 8-16$.

7-.Palframan SL, Kwok T, Gabriel K. Vacuolatingcytotoxin A (VacA), a key toxin for Helicobacter pylori pathogenesisFrontCell InfectMicrobiol. 2012;2:92.

8-. Foegeding NJ, Caston RR, McClain MS, Ohi MD, Cover TL. An overview of Helicobacter pylori VacA toxin biology. Toxins (Basel). 2016;8(6). pii: E173.

9-.Kononov AV. [Inflammation as abasisforHelicobacterpylor-associated diseases].

10-Lovell RM, Ford AC. Effect of gender on prevalence of irritable bowel syndrome in the community: systematic review and meta-analysis. Am J Gastroenterol. 2012;107(7):9911000.

11-.Hungin AP, Whorwell PJ, Tack J, Mearin F. The prevalence, patterns and impact of irritable bowel syndrome: an international 
